\title{
CHUVAS EXTREMAS EM ALEGRETE (RS): UM ESTUDO SOBRE SUA OCORRÊNCIA NO SÉCULO XX
}

\author{
EXTREME RAINFALL IN ALEGRETE (RS): A STUDY ON \\ THE OCCURRENCE IN THE TWENTIETH CENTURY \\ PRECIPITACIÓN EXTREMA EN ALEGRETE (RS): UN \\ ESTUDIO SOBRE LA OCURRENCIA EN EL SIGLO XX
}

Fabio de Oliveira Sanches - Universidade Federal do Triângulo Mineiro - Uberaba - Minas Gerais - Brasil fsanches.73@gmail.com
Roberto Verdum - Universidade Federal do Rio Grande do Sul - Porto Alegre - Rio Grande do Sul - Brasil verdum@ufrgs.br

Gilberto Fisch - Universidade de Taubaté - Taubaté - São Paulo - Brasil
fisch.gilberto@gmail.com

\begin{abstract}
Resumo
Informações do $5^{\circ}$ Relatório de Avaliação das Mudanças do Clima do Planeta (AR5) do Painel Intergovernamental de Mudanças Climáticas (IPCC) afirmam que os eventos extremos de precipitação se tornarão mais intensos e frequentes até o final do século XXI. Neste trabalho objetiva-se identificar as frequências, absoluta e relativa, dos eventos intensos de precipitação e seu tempo de retorno, para a região no entorno dos postos meteorológicos de Alegrete (RS) no século XX. Foram utilizados dados pluviométricos diários dos postos da Agência Nacional de Águas (ANA) no período de 1928-2009. Os resultados indicaram que os dias com chuvas entre 50 e $80 \mathrm{~mm}$ são comuns no ano todo. Dias com chuvas $\geq 100 \mathrm{~mm}$ ocorrem uma vez ao ano. Dias com chuvas $\geq 150 \mathrm{~mm}, \geq 160 \mathrm{~mm}, \geq 170 \mathrm{~mm}$ e $\geq 180 \mathrm{~mm}$ apresentam, respectivamente, tempo de retorno de 8 , 21, 27 e 82 anos. Esses eventos intensos são responsáveis por parte da dinâmica da arenização em Alegrete (RS).
\end{abstract}

Palavras-chave: arenização, chuvas extremas, mudanças climáticas, sudoeste do Rio Grande do Sul.

\section{Abstract}

The 5th Assessment Report of the Earth Climate Change (AR5) of the Intergovernmental Panel on Climate Change (IPCC) says that extreme precipitation events will become more intense and frequent until the end of the XXI century. This study aims to identify the frequency, absolute and relative, of intense precipitation events and their time of return for the surrounding region of meteorological stations of Alegrete (RS) in the twentieth century. Daily rainfall data from stations of the Agência Nacional de Águas (ANA) in the period of 1928-2009 were used in this study. The results indicated that rainy days with between $50 \mathrm{~mm}$ and $80 \mathrm{~mm}$ are common every year. Days with rainfall $\geq 100 \mathrm{~mm}$ occur once a year. Days with rainfall $\geq 150 \mathrm{~mm}, \geq 160 \mathrm{~mm}$, $\geq 170 \mathrm{~mm}$ and $\geq 180 \mathrm{~mm}$ respectively show recurrence time of 8 years, 21 years, 27 years and 82 years. These intense events are responsible for part of the dynamics of sandization in Alegrete (RS).

Keywords: sandization, extreme rainfall, climate change, southwest of Rio Grande do Sul. 


\section{Resumen}

Informaciones del $5^{0}$ Informe de Evaluación de los Cambios del Clima del Planeta (AR5) del Grupo Intergubernamental de Expertos sobre el Cambio Climático (IPCC) afirman que los eventos extremos de precipitación se harán más intensos y frecuentes hasta el final del siglo XXI. Este estudio tiene como objetivo identificar las frecuencias, absoluta y relativa, de las precipitaciones intensas y su tiempo de retorno, para la región de Alegrete (RS) en el siglo XX. Se utilizaron los datos de precipitación diaria de las estaciones de la Agencia Nacional de Aguas (ANA) en el período de 1928-2009. Los resultados mostraron que los días de lluvia entre $50 \mathrm{~mm}$ y $80 \mathrm{~mm}$ son comunes todo el año. Días con lluvias $\geq 100 \mathrm{~mm}$ ocurren una vez al año. Días con lluvias $\geq 150 \mathrm{~mm}, \geq 160 \mathrm{~mm}, \geq 170 \mathrm{~mm}$ y $\geq 180 \mathrm{~mm}$, presentan, respectivamente, tiempo de retorno de 8 años, 21 años, 27 años y 82 años. Esos eventos intensos son responsables por parte de la dinámica de arenización en Alegrete (RS).

Palabras-clave: arenización, lluvias extremas, cambio climático, suroeste de Rio Grande do Sul.

\section{Introdução}

A publicação do $5^{\circ}$ Relatório de Avaliação das Mudanças do Clima do Planeta (AR5), organizado pelo Intergovernmental Panel on Climate Change (IPCC, 2013), em setembro de 2013, reforçou as evidências publicadas anteriormente (AR4) sobre as mudanças climáticas.

Com base em análises científicas, o AR5 apontou, como inequívoco, o aquecimento do sistema climático, além de afirmar que, desde a década de 1950, as mudanças climáticas são sem precedentes (aumento das temperaturas da atmosfera e dos oceanos, diminuição das superfícies de gelo e neve, aumento do nível médio dos oceanos e aumento nas concentrações de gases do efeito estufa) (IPCC, 2013).

O AR5 também reforçou a ideia de que, com o aumento da temperatura média da superfície global, os eventos extremos de precipitação sobre os continentes, em latitudes médias e regiões tropicais, muito provavelmente, se tornarão mais intensos e frequentes até o final do século XXI (IPCC, 2013).

Sob essa perspectiva, Marengo (2007) acredita que as evidências de tais mudanças poderiam afetar significativamente o planeta, de maneira muito mais rigorosa nos países menos desenvolvidos situados na região tropical, especialmente em relação à ocorrência de extremos climáticos. Nesse contexto, o autor ressalta que o Brasil pode tornar-se muito vulnerável às mudanças climáticas atuais e, mais ainda, às mudanças que se projetam para o futuro, sobretudo aquelas associadas aos eventos climáticos intensos.

Para Mendonça (2006), os prognósticos contidos nos relatórios do IPCC consideram que as variações dos componentes do ciclo hidrológico, destacando a precipitação e a evapotranspiração, irão atuar, de forma 
muito diferenciada, sobre a distribuição de água no planeta, levando algumas regiões a terem um incremento em seus volumes de água e, dessa forma, intensificando a ocorrência de chuvas torrenciais, enchentes, deslizamentos, movimentos de massa e processos erosivos.

Considerando a ocorrência de processos erosivos na porção sudoeste do estado do Rio Grande do Sul, em meio à formação campestre do bioma Pampa, existem áreas com solos arenosos, descobertos e com grande potencial de fragilidade, conhecidos localmente por areais.

Verdum (1997; 2004) desenvolveu análises sobre a dinâmica hidroclimática nos processos morfoesculturais na região dos areais e comprovou que as chuvas irregulares e, por vezes, torrenciais são responsáveis pela formação de ravinas e voçorocas.

Ao analisar dados de precipitação diária, no decorrer do século XX, para a região de Alegrete, Sancheset al. (2014) identificou que as chuvas diárias de forte intensidade (entre 80 e $100 \mathrm{~mm}$ ), respectivamente nos meses de novembro e fevereiro, apresentaram tendência significativa de aumento, caracterizando-se como evidência de mudanças climáticas.

Dessa forma, associando os estudos desenvolvidos por Suertegaray (1987; 2012), Suertegaray et al. (2001), Verdum (1997; 2004; 2012) com o de Sanches (2013) e Sanches et al. (2014) é possível considerar que o aumento na ocorrência desses eventos de pluviosidade elevada possa intensificar a dinâmica da água nesses solos (arenosos) acelerando processos geomorfológicos superficiais concentrados, os quais originam sulcos, ravinas e voçorocas.

Dessa forma, identificar, com detalhes, a ocorrência de eventos de pluviosidade extrema permite compreender os processos geomorfológicos responsáveis pela dinâmica da arenização no Rio Grande do Sul.

Assim sendo, o objetivo deste trabalho consiste em identificarra frequência (absoluta e relativa) dos eventos intensos de precipitação, bem como o tempo de retorno desses eventos no decorrer do século XX, em Alegrete (RS). Estes resultados possuem aplicação prática na questão ambiental da temática da arenização.

\section{Material e Método}

Para o desenvolvimento deste trabalho foram utilizados dados pluviométricos diários obtidos da rede de postos da Agência Nacional de Águas (ANA), disponíveis por meio da plataforma eletrônica Hidroweb, 
sendo selecionadas informações dos postos de Alegrete (INMET), Alegrete (ANA), Passo Mariano Pinto, Fazenda Três Capões e Estação do Tigre (Figura 1).

Figura 1 - Localização dos postos pluviométricos utilizados em Alegrete (RS).

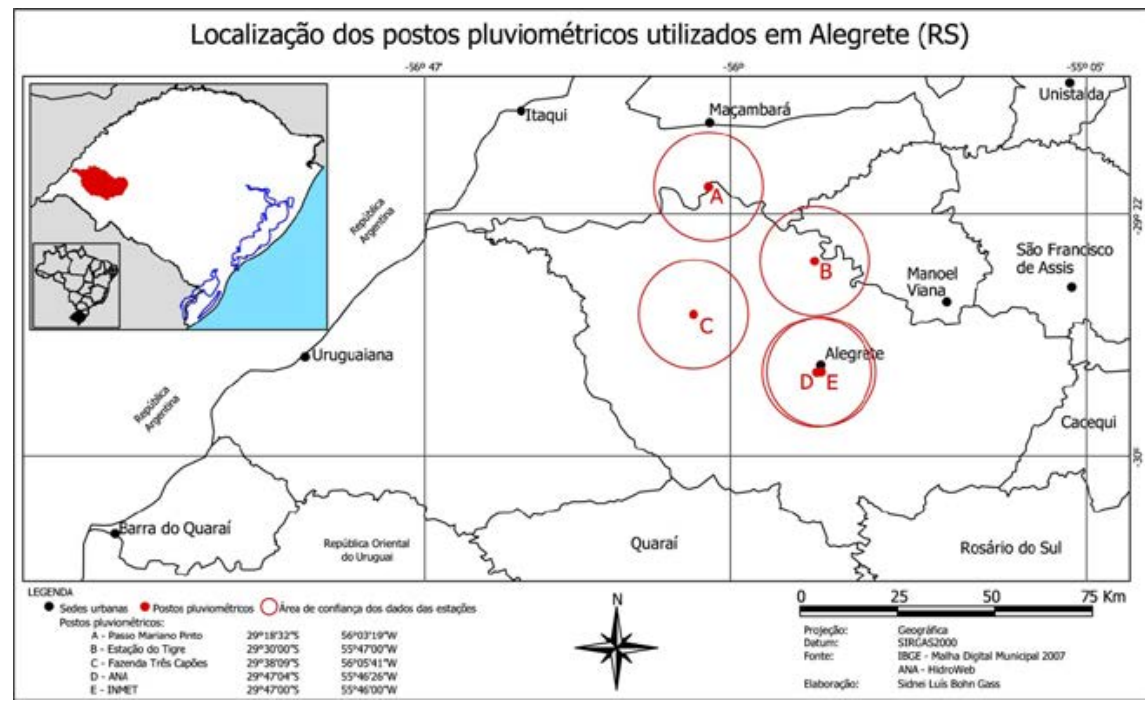

Fonte: Sanches (2013)

Na busca por obter uma série contínua e homogênea de dados, procurou-se adotar como postos principais, inicialmente, os postos de Alegrete (INMET) e Passo Mariano Pinto, por apresentarem os maiores conjuntos contínuos de dados diários.

A etapa consistiu em preencher as falhas dos conjuntos de dados com as informações dos demais postos selecionados. Testes estatísticos como Correlação de Pearson, Regressão Linear e Teste de Dupla Massa foram aplicados aos dados de períodos comuns como forma de garantir a confiabilidade estatística no preenchimento das falhas.

Como a literatura que aborda a dinâmica da arenização no sudoeste gaúcho destaca que os processos de escoamento superficial estão vinculados aos eventos diários de precipitação intensa (Verdum, 1997; Suertegarayet al., 2001 e Verdum, 2012), a etapa posterior possibilitou identificar, no decorrer de toda a série de dados diários, a quantidade de dias com precipitações $\geq 50 \mathrm{~mm}, \geq 80 \mathrm{~mm}$ e $\geq 100 \mathrm{~mm}$, para a análise de ocorrência no decorrer do século XX. 
Dessa forma, os valores pluviométricos diários da série de Alegrete (1928-2009) foram dispostos em um gráfico para análise (Figura 2). A partir dessas informações foi possível estabelecer a quantidade $(f)$ de eventos pluviométricos $\geq 50 \mathrm{~mm}, \geq 80 \mathrm{~mm}, \geq 100 \mathrm{~mm}, \geq 150 \mathrm{~mm}, \geq$ $160 \mathrm{~mm}, \geq 170 \mathrm{~mm} \mathrm{e} \geq 180 \mathrm{~mm}$.

O passo seguinte consistiu em determinar a frequência relativa $(f r)$ para cada evento extremo, por meio da seguinte fórmula, como sugere Galvani (2011).

$$
\mathrm{fr}=\frac{\mathrm{na}}{n}
$$

A frequência relativa $(f r)$ corresponde ao número de vezes que o evento ocorreu (na) em relação ao número total de elementos da série (n). No caso dos dados de Alegrete (1928-2009), a quantidade de cada evento pluviométrico extremo é em função dos 82 anos da série.

De posse de tais informações, o passo seguinte foi determinar o tempo de retorno para cada evento extremo de precipitação, sendo este definido como o inverso da probabilidade (Tucci, 2009; Galvani, 2011). O objetivo é determinar qual é o período de retorno para um evento de precipitação extrema.

$$
\mathrm{T}=\frac{1}{\mathrm{fr}}
$$

Desse modo, com base na frequência (quantidade dos eventos), na frequência relativa ( $f r$ ) e no seu tempo de retorno $(T)$ foi possível verificar quais foram às dinâmicas desses eventos extremos no decorrer do século XX.

\section{Resultados e Discussão}

O preenchimento das falhas nas séries de dados de Alegrete (INMET) e Passo Mariano Pinto teve início aplicando-se a Correlação de Pearson entre dados mensais de precipitação comuns aos postos utilizados (Tabela 1). A partir de seus elevados coeficientes de determinação $\left(R^{2}\right)$ e de correlação $(r)$ foi possível aceitar a substituição dos dados faltantes nos postos de Alegrete (INMET) e Passo Mariano Pinto, pelos dados correspondentes aos demais postos utilizados no trabalho. 
Tabela 1 - Relação dos postos correlacionados para preenchimento de falhas por Regressão Linear

\begin{tabular}{ccc}
\hline Postos correlacionados & Período & Coeficientes R $\mathbf{R}^{2}$ r \\
\hline Alegrete (INMET) e Faz. 3 Capões & $\begin{array}{c}1953,1954,1955,1956,1957,1958,1959 \\
\text { e 1960. }\end{array}$ & $\begin{array}{c}\mathrm{R}^{2}=0,9924 \\
\mathrm{r}=0,9961\end{array}$ \\
\hline Alegrete (INMET) e Estação do Tigre & 1944 e 1953. & $\begin{array}{c}\mathrm{R}^{2}=0,9795 \\
\mathrm{r}=0,9896\end{array}$ \\
\hline Alegrete (INMET) e Passo Mariano Pinto & $1975,1977,1978,1979,1980,1981,1983$, & $\begin{array}{c}\mathrm{R}^{2}=0,9884 \\
\mathrm{r}=0,9941\end{array}$ \\
\hline \multirow{2}{*}{ Alegrete (ANA) e Passo Mariano Pinto } & 1984 e 1985. & $\begin{array}{c}\mathrm{R}^{2}=0,9799 \\
\mathrm{r}=0,9898\end{array}$ \\
\hline
\end{tabular}

Dessa forma, o Quadro 1 apresenta a sequência temporal dos postos pluviométricos utilizados no trabalho para a obtenção de uma série contínua de dados.

Quadro 1 - Sequência temporal de dados pluviométricos para a série de Alegrete (RS)

\begin{tabular}{|c|c|}
\hline Posto Pluviométrico & Período \\
\hline Alegrete (INMET) & $1928-1946$ \\
\hline Estação do Tigre & $1947-1952$ \\
\hline Alegrete (INMET) & $1953-1969$ \\
\hline Passo Mariano Pinto & $1970-1978$ \\
\hline Alegrete (INMET) & $1979-1985$ \\
\hline Passo Mariano Pinto & 1986 \\
\hline Alegrete (ANA) & $1987-1995$ \\
\hline Passo Mariano Pinto & $1996-2009$ \\
\hline
\end{tabular}

Conforme recomenda Tucci (2009), a Figura 2 apresenta a Curva de Dupla Massa obtida para a verificação de consistência entre os dados da série temporal de Alegrete (1928-2009) e as Normais Climatológicas (19311960 e 1961-1990) do posto meteorológico de Uruguaiana, localizado no bioma Pampa, na mesma faixa latitudinal e distante, aproximadamente, $126 \mathrm{~km}$ do posto Alegrete (INMET).

A Figura 2 demonstra o elevado grau de homogeneidade (99,6\%) entre a série temporal de Alegrete (1928-2009) em conjunto com as Normais Climatológicas (1931-1960 e 1961-1990) de Uruguaiana, por meio do método de Dupla Massa. 
Figura 2 - Curva de Dupla Massa para análise de consistência entre os dados de Alegrete (RS) e as Normas Climatológicas (1931-1960 e 1961-1990) de Uruguaiana (RS)

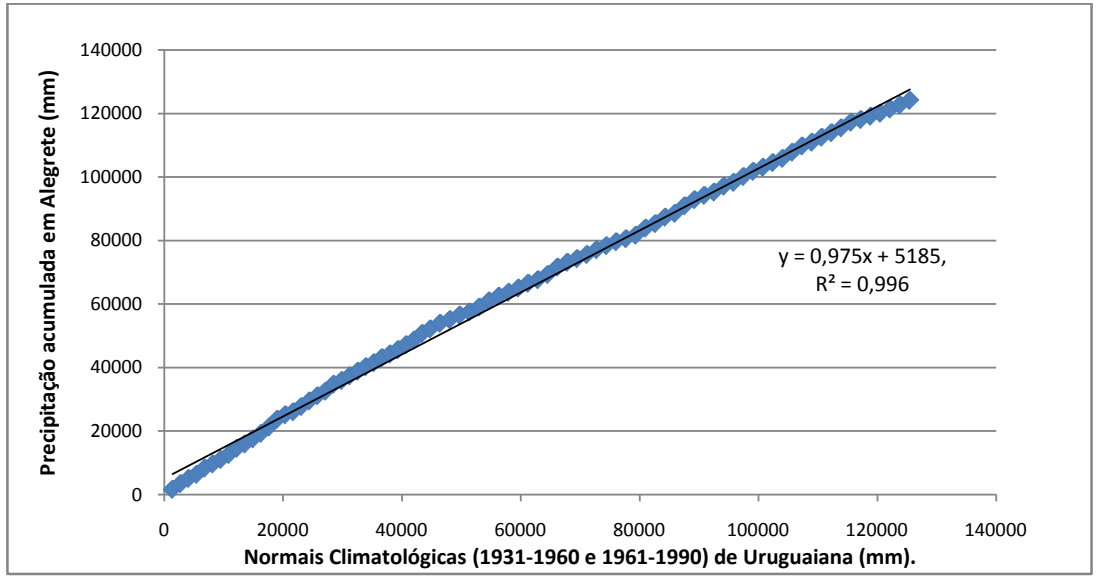

Fonte: Sanches (2013)

O alinhamento desses pontos sobre a reta de regressão linear sugere uma boa consistência do conjunto de dados, descartando a possibilidade de haver erros sistemáticos, alteração nas condições de observação e possíveis mudanças nas condições climáticas regionais.

Assim sendo, ao término do processo de preparação dos dados dos postos pluviométricos do município de Alegrete, obteve-se um conjunto de dados diários de precipitação para a série temporal de Alegrete (19282009) a ser analisado.

O estudo de uma longa série temporal de precipitação como a de Alegrete (1928-2009) permite que sejam analisadas, além de suas variabilidades, tendências e padrões, a frequência com que os eventos ocorreram no decorrer do período de estudo, sobretudo os eventos extremos (chuvas extremas, por exemplo).

A Figura 3 apresenta a sequência e os valores das precipitações diárias no decorrer dos 82 anos da série de Alegrete (1928-2009). Uma análise preliminar revela que os eventos pluviométricos acima de $50 \mathrm{~mm}$ foram comuns no decorrer de todo o conjunto de dados analisados. Claramente, também, se observam valores pluviométricos extremos que são superiores a $100 \mathrm{~mm}$, ao longo de toda a série. Entretanto, uma análise mais sistemática permite que se calcule a quantidade de eventos de precipitação extrema $(f)$, sua frequência relativa $(f r)$ e seu tempo de retorno $(T)$. 
Figura 3 - Série temporal das precipitações diárias da série de Alegrete (1928-2009)

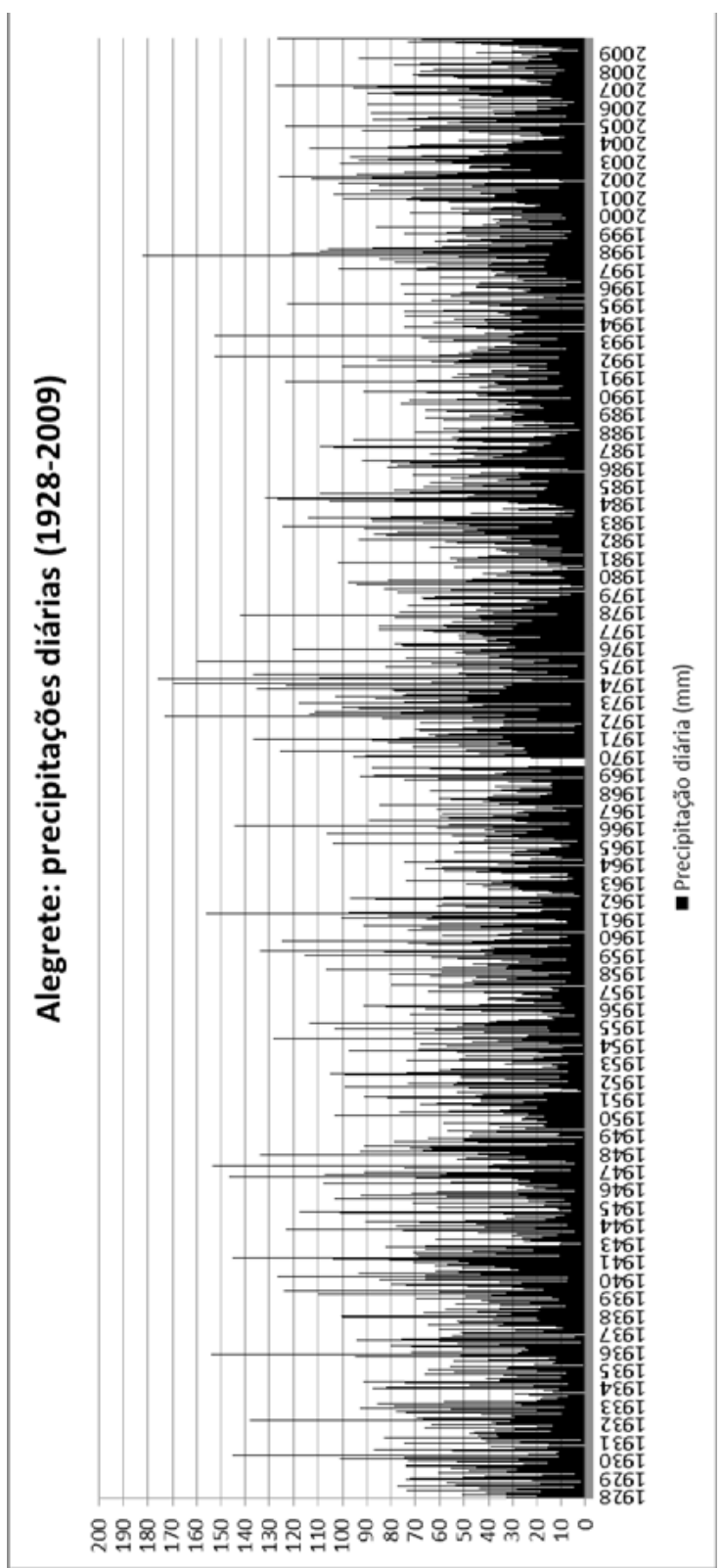

Fonte: Sanches (2013) 
A organização dos dados pluviométricos diários possibilitou observar a quantidade (frequência) de eventos de chuva intensa, bem como determinar a frequência relativa $(f r)$ e o tempo de retorno $(T)$ dos mesmos (Tabela 2).

Verifica-se assim, por meio da Tabela 2, que a elevada frequência de eventos de precipitação diária $\geq 50 \mathrm{~mm}$ possibilita uma frequência relativa de cerca de oito dias ao ano com precipitações dessa intensidade, no decorrer dos 82 anos de dados analisados.

Os 183 eventos diários de precipitações $\geq 80 \mathrm{~mm}$ observados em toda a série revelam uma frequência relativa de cerca de, ao menos, dois desses eventos a cada ano.

Em relação às chuvas diárias com valores $\geq 100 \mathrm{~mm}$, foram observados 84 eventos em toda a série analisada. Dessa forma, a possibilidade de ocorrerem eventos dessa intensidade é de, ao menos, um evento a cada ano, como aponta o cálculo do seu tempo de retorno (Tabela 2).

Tabela 2 - Cálculo da Frequência, Frequência Relativa e do Tempo de Retorno dos eventos intensos de precipitação para a série de Alegrete (1928-2009)

\begin{tabular}{cccc}
\hline Eventos & $\begin{array}{c}\text { Frequência } \\
\text { (Quant. eventos) }\end{array}$ & $\begin{array}{c}\text { Frequência relativa } \\
\text { (Quant. eventos/ano) }\end{array}$ & $\begin{array}{c}\text { Tempo de retorno } \\
\text { (anos) }\end{array}$ \\
\hline$\geq 50 \mathrm{~mm}$ & 648 & 7,90 & 0,12 (7,9 dias/ano) \\
$\geq 80 \mathrm{~mm}$ & 183 & 2,23 & 0,44 (2,3 dias/ano) \\
$\geq 100 \mathrm{~mm}$ & 84 & 1,02 & 1 \\
$\geq 150 \mathrm{~mm}$ & 10 & 0,12 & 8 \\
$\geq 160 \mathrm{~mm}$ & 4 & 0,05 & 21 \\
$\geq 170 \mathrm{~mm}$ & 3 & 0,04 & 27 \\
$\geq 180 \mathrm{~mm}$ & 1 & 0,01 & 82 \\
\hline
\end{tabular}

Já eventos de maior intensidade também foram observados nos 82 anos da série. Precipitações diárias $\geq 150 \mathrm{~mm}, \geq 160 \mathrm{~mm}, \geq 170 \mathrm{~mm}$ e $\geq 180 \mathrm{~mm}$ tiveram seu tempo de retorno calculado em função da sua probabilidade, sendo eles, respectivamente, de 8, 21, 27 e 82 anos.

Dessa forma, uma vez que as precipitações extremas são as responsáveis por processos de ravinamentos e voçorocamentos na dinâmica da arenização, observa-se, com base nas informações da Tabela 2, que tais eventos, sobretudo os $\geq 100 \mathrm{~mm}$ ao ano, foram comuns anualmente durante praticamente todo o século XX. 
Um aspecto que merece destaque consiste nos resultados encontrados por Sanches (2013), o qual, analisando a dinâmica das chuvas diárias no século XX e suas relações com as mudanças climáticas, afirma que as precipitações intensas, sobretudo as superiores a $100 \mathrm{~mm} / \mathrm{dia}$, tornaram-se mais frequentes no decorrer do século XX. Tais resultados podem ser considerados como evidências de mudanças climáticas, os quais intensificaram os processos de escoamento superficial associados à dinâmica erosiva.

Dessa forma, considerando os trabalhos de Suertegaray (1987; 2012), Suertegaray et al. (2001) e Verdum (1997; 2004; 2012), que analisaram a dinâmica dos areais do sudoeste do Rio Grande do Sul associando-as, ao menos em parte, ao comportamento das precipitações intensas e por vezes concentradas, é possível afirmar que as precipitações intensas, com volumes diários superiores a $50 \mathrm{~mm}$, foram (e são) responsáveis pela intensificação do escoamento superficial, sobretudo o escoamento concentrado, o qual se destaca na dinâmica erosiva da arenização.

\section{Considerações Finais}

Para Marengo (2007), no Brasil, são raros os estudos sobre a variabilidade climática de longo prazo e seus eventos extremos. A falta de informações meteorológicas, a baixa confiabilidade dos dados e o desenvolvimento de diferentes metodologias caracterizam os estudos existentes.

Dessa forma, a reconstrução de uma série de dados pluviométricos diários para a região no entorno do município de Alegrete (RS) pode ser considerada um resultado que agrega informações na questão de mudanças climáticas. A seleção dos postos pluviométricos, a adoção de métodos e técnicas estatísticas para preenchimento de falhas e a verificação de sua consistência forneceram a confiabilidade técnica para a análise seguinte.

A análise de uma série de 82 anos com eventos pluviométricos diários permitiu observar que os dias com precipitações entre 50 e 80 $\mathrm{mm}$ foram anualmente frequentes no período de 1928-2009. Já os dias com precipitações superiores a $100 \mathrm{~mm}$ foram recorrentes, ao menos, uma vez por ano.

Esses resultados reforçam as pesquisas de Suertegaray (1987 e 2012), Suertegaray et al. (2001) e Verdum (1997; 2004; 2012), sobre parte da 
dinâmica da arenização no sudoeste gaúcho decorrer do comportamento das precipitações intensas e, por vezes, concentradas.

A série revelou, ainda, que eventos pluviométricos diários superiores a $150 \mathrm{~mm}$ ( $\geq 150 \mathrm{~mm}, \geq 160 \mathrm{~mm}, \geq 170 \mathrm{~mm}$ e $\geq 180 \mathrm{~mm}$ ) contribuíram de forma a intensificar a dinâmica da arenização no sudoeste gaúcho, porém, com um tempo de recorrência menor, respectivamente, 8, 21, 27 e 82 anos.

Assim sendo, os resultados encontrados neste trabalho demonstraram que os eventos intensos de precipitação diária foram fortemente recorrentes no decorrer do século XX, influenciando, significativamente, na dinâmica geomorfológica superficial dos areais no sudoeste do Rio Grande do Sul.

\section{Agradecimentos}

Os autores agradecem à FAPERGS (Edital 04/2012 - PQ Gaúcho), ao Dr. José Marengo e à FAPESP (através do processo 2008/58161-1) e ao CNPQ (MCTI/CNPQ - Universal n. 14/2012) pelo auxílio no desenvolvimento da pesquisa.

\section{Referências}

GALVANI, E. Estatística descritiva em sala de aula. In: VENTURI, L.A.B. Geografia: práticas de campo, laboratório e sala de aula. São Paulo: Ed. Sarandi, 2011.

IPCC.Technical Summary. In: INTERGOVERNMENTAL PANEL ON CLIMATE CHANGE. Contribution of Working Group I to the Fifth Assessment Report. Climate change 2013: the physical science basis.Cambridge/New York:Cambridge University Press, 2013. Disponível em: <http://www.ipcc.ch/report/ar5/wg1/>.

MARENGO, J.A. Caracterização do clima no século XX e cenários no Brasil e na América do Sul para o século XXI derivados dos Modelos de Clima do IPCC. Relatório $\mathrm{n}^{\mathrm{o}}$ 1. Mudanças climáticas globais e seus efeitos sobre a diversidade. MMA. São Paulo: CPTEC/INPE, 2007. Disponível em:<http:// mudancasclimaticas.cptec.inpe.br/ $\sim \mathrm{rmclima} / \mathrm{pdfs} /$ prod_probio/Relatorio_1. pdf $>$. Acesso em: 12 maio 2011.

MENDONÇA, F. Aquecimento global e suas manifestações regionais e locais: alguns indicadores da Região Sul do Brasil. Revista Brasileira de Climatologia, v. 2, p. 71-86, 2006.Disponível em: <http://ojs.c3sl.ufpr.br/ojs2/index.php/ revistaabclima/article/viewFile/25388/17013>. Acesso em: 15 jan. 2011.

SANCHES, F. O. Os areais do sudoeste do Rio Grande do Sul: estudo sobre as chuvas no século XX e um possível cenário para o século XXI. Tese (doutorado). 
Programa de Pós-Graduação em Geografia, Universidade Federal do Rio Grande do Sul, Porto Alegre, 2013.

SANCHES, F. O.; VERDUM, R.; FISCH, G. Tendência de longo prazo das chuvas diárias no Sudoeste do Rio Grande do Sul: os eventos extremos e a arenização. Revista Brasileira de Geografia Física, v.7, n.6, p. 1100-1109, 2014. Disponível em:<http://www.revista.ufpe.br/rbgfe/index.php/revista/article/view/799/645>. Acesso em: 10 jan. 2015.

SUERTEGARAY, D.M.A. A trajetória da natureza: um estudo geomorfológico sobre os areais de Quarai - RS. Tese (doutorado em Geografia). Faculdade de Filosofia, Letras e Ciências Humanas, Universidade de São Paulo, São Paulo, 1987.

SUERTEGARAY, D. M. A. Arenização: análise morfogênica. In: SUERTEGARAY, D. M. A.; PIRES DA SILVA, L. A.; GUASSELLI, L. A. (Org.). Arenização: natureza socializada. Porto Alegre: Compasso Lugar-Cultura/Imprensa Livre, 2012.

SUERTEGARAY, D.M.A.; GUASSELLI, L.A.; VERDUM, R. (Org.). Atlas da Arenizaçãono Sudoeste do Rio Grande do Sul. Porto Alegre: Secretaria da Coordenação e Planejamento e Secretaria da Ciência e Tecnologia do Governo do Estado do Rio Grande do Sul, 2001. Disponível em:<http://www.ufrgs.br/areais. pampa/atlas.htm>. Acesso em: 10 maio 2011.

TUCCI, C.E.M. (Org.) Hidrologia: ciência de aplicação.4.ed., 1.reimp. Porto Alegre: Editora da UFRGS/ABRH, 2009.

VERDUM, R. Approche géographique des "déserts" dans les communes de São Francisco de Assis et Manuel Viana, État du Rio Grande do Sul, Brésil. Tese (doutorado). UFR de Géographie et Aménagement, Université de Toulouse Le Mirail, Toulouse, 1997.

VERDUM, R. Depressão periférica e planalto. Potencial ecológico e utilização social da natureza. In: VERDUM, R.; BASSO, L. A.; SUERTEGARAY, D. M. A. (Org.). Rio Grande do Sul: paisagens e territórios em transformação. Porto Alegre: Editora da UFRGS, 2004.

VERDUM, R. Descoberta permanente: das areias aos areais. In: SUERTEGARAY, D.M.A.; PIRES DA SILVA, L. A.; GUASSELLI, L.A. Arenização: natureza socializada. Porto Alegre: Compasso Lugar-Cultura/Imprensa Livre, 2012.

Fabio de Oliveira Sanches -Possui Graduação em Geografia pela Universidade de Sorocaba. Mestrado em Ciências Ambientais pela Universidade de Taubaté. Possui Doutorado em Geografia pela Universidade Federal do Rio Grande do Sul. Atualmente é professor pela Universidade Federal do Triângulo Mineiro.

Roberto Verdum - Possui graduação em Geografia pela Universidade Federal do Rio Grande do Sul. Mestrado em Géographie et Aménagement e Doutorado em Géographie et Aménagement pela Université de Toulouse Le Mirail, França. Atualmente é Professor Associado do Departamento de Geografia Universidade Federal do Rio Grande do Sul. 
Gilberto Fisch - Possui Graduação em Meteorologia pela Universidade de São Paulo e Mestrado em Meteorologia pela mesma Universidade. Possui Doutorado em Meteorologia pelo Instituto Nacional de Pesquisas Espaciais. Atualmente é pesquisador titular do Instituto de Aeronaútica e Espaço e Professor da Universidade de Taubaté.

Contribuição de cada autor no desenvolvimento do artigo:

Todos os autores ofereceram substanciais contribuições científicas e intelectuais ao desenvolvimento do artigo. Este consiste em um subproduto da tese de doutorado do primeiro autor, sob orientação do segundo e terceiro autores. Coube ao segundo autor a redação da fundamentação teórica-conceitual referente ao processo de arenização. Ao terceiro autor, coube o desenvolvimento das técnicas de análise para a reconstrução estatística do fenômeno estudado. As demais partes do artigo ficaram sob responsabilidade do primeiro autor.

Recebido para publicação em 20 de maio de 2016

Aceito para publicação em 11 de junho de 2016 
